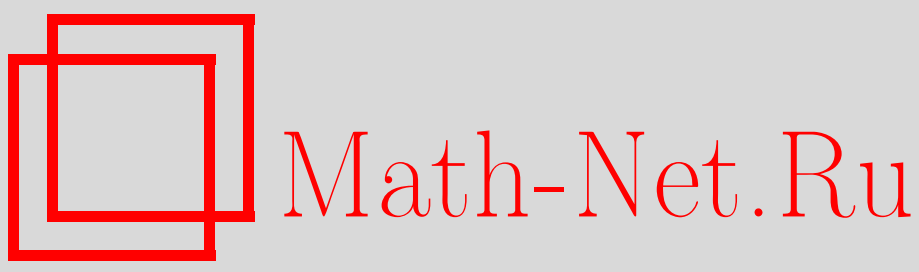

Вик. С. Куликов, Конечная определенность коммутанта фундаментальной группы дополнения к плоской кривой, Изв. РАН. Сер. матем., 1997, том 61, выпуск 5, 6370

DOI: https://doi.org/10.4213/im151

Использование Общероссийского математического портала Math-Net.Ru подразумевает, что вы прочитали и согласны с пользовательским соглашением

http://www . mathnet.ru/rus/agreement

Параметры загрузки:

IP : 54.174 .149 .18

26 апреля 2023 г., 12:47:46 
УДК $512.7+515.1$

Вик. С. Куликов

\title{
Конечная определенность коммутанта фундаментальной группы дополнения к плоской кривой
}

\author{
В статье доказана следующая теорема. Коммутант фундаментальной группы \\ дополнения к плоской неприводимой проективной кривой является конечно опре- \\ деленной группой. \\ Библиографиия: 3 наименования.
}

0. Пусть $\bar{D} \subset \mathbb{P}^{2}$ - проективная алгебраическая кривая, определенная над полем комплексных чисел $\mathbb{C}$. Обозначим через $\bar{G}=\pi_{1}\left(\mathbb{P}^{2} \backslash \bar{D}\right)$ фундаментальную группу дополнения к кривой $\bar{D}$ в $\mathbb{P}^{2}$.

Как действительное многообразие $\bar{D}$ является подмногообразием действительной коразмерности 2 в $\mathbb{P}^{2}$. Эта ситуация аналогична ситуации в теории узлов: узел $k$ является подмногообразием действительной коразмерности 2 в трехмерной сфере $S^{3}$. Обозначим через $G=\pi_{1}\left(S^{3} \backslash k\right)$ группу узла $k$ и через $N=[G, G]$ ее коммутант. Хорошо известно, что множество узлов распадается на два подмножества в соответствии со свойствами их груп,, а именно, по теореме Столлингса [3] $N$ является конечно определенной группой тогда и только тогда, когда $k$ - расслоенньй узел, т.е. $S^{3} \backslash k$ допускает структуру расслоения над $S^{1}$ с поверхностями Зайферта в качестве слоев.

Цель этой короткой заметки - доказать следующую теорему.

ТЕОРема 1. Если $\bar{D} \subset \mathbb{P}^{2}$ - неприводимая проективная кривая, то коммутант $\bar{N}=[\bar{G}, \bar{G}]$ группь $\bar{G}=\pi_{1}\left(\mathbb{P}^{2} \backslash \bar{D}\right)$ является конечно определенной əpynпой.

Эта теорема является простым следствием следующего аналога этой теоремы для случая аффинной кривой.

ТЕОРема 2. Если $D \subset \mathbb{C}^{2}$ - аффинная неприводимая кривая такая, что ее проективное замыкание $\bar{D} \subset \mathbb{P}^{2}$ и прямая в бесконечности $L_{\infty}=\mathbb{P}^{2} \backslash \mathbb{C}^{2}$ пересекаются трансверсально, то коммутант $N=[G, G]$ әруппьи $G=\pi_{1}\left(\mathbb{C}^{2} \backslash D\right)$ является конечно определенной группой.

Работа была выполнена при частичной финансовой поддержке фонда INTAS (грант № 94-4373) и РФФИ (грант № 96-01-00614).

(C) Вик.С. Куликов 1997 
В [1] уже было доказано, что коммутант $N=[G, G]$ групшы $G=\pi_{1}\left(\mathbb{C}^{2} \backslash D\right)$ конечно порожден для любой неприводимой аффинной кривой. Доказывая теоремы 1 и 2 , мы будем существенно опираться на идеи и результаты из [1].

Ниже мы рассмотрим более общую ситуацию, когда $\bar{D}=\bar{D}_{1}+\cdots+\bar{D}_{n}$ является приведенной приводимой кривой.

Пусть $L \subset \mathbb{P}^{2}$ - прямая. Положим $L=L_{\infty}, \mathbb{C}^{2}=\mathbb{P}^{2} \backslash L_{\infty}, D_{i}=\bar{D}_{i} \cap \mathbb{C}^{2}$, и пусть $f_{i}(x, y)=0$ - уравнение $D_{i}$, где $f_{i}(x, y) \in \mathbb{C}[x, y]$ - неприводимый многочлен.

Обозначим через

$$
F: X=\mathbb{C}^{2} \backslash D \rightarrow \mathbb{C}^{*}=\mathbb{C} \backslash\{0\}
$$

морфизм, заданный уравнением

$$
z=\prod_{i=1}^{n} f_{i}(x, y)
$$

Мы будем предполагать, что выполнено следуюшее условие:

$$
\text { общий слой } F^{-1}(z)=Y_{z} \text { связен. }
$$

Отметим, что если $D$ связно в $\mathbb{C}^{2}$, то $F$ удовлетворяет условию $(*)$.

Teорема $2^{\prime}$. Если $\bar{D}=\bar{D}_{1}+\cdots+\bar{D}_{n} \subset \mathbb{P}^{2}$ и $L_{\infty}$ пересекаются трансверсально и $D$ удовлетворяет условию $(*)$, то ядро $N$ индуцированного гомоморфизма $F_{*}: \pi_{1}\left(\mathbb{C}^{2} \backslash D\right) \rightarrow \pi_{1}\left(\mathbb{C}^{*}\right)$ является конечно определенной группой.

Теорема 2 является следствием теоремы $2^{\prime}$, так как если $D$ - неприводимая кривая, то ker $F_{*}$ совпадает с коммутантом группы $\pi_{1}\left(\mathbb{C}^{2} \backslash \bar{D}\right)$.

1. Теорема 1 следует из теоремы 2. Действительно, рассмотрим неприводимую проективную кривую $\bar{D} \subset \mathbb{P}^{2}$ и выберем бесконечно удаленную прямую $L_{\infty} \subset \mathbb{P}^{2}$ так, что $\bar{D}$ и $L_{\infty}$ пересекаются трансверсально. Имеем естественньй гомоморфизм

$$
i_{*}: G=\pi_{1}\left(\mathbb{C}^{2} \backslash D\right) \rightarrow \bar{G}=\pi_{1}\left(\mathbb{P}^{2} \backslash \bar{D}\right)
$$

индуцированный вложением $i: \mathbb{C}^{2} \backslash D \rightarrow \mathbb{P}^{2} \backslash \bar{D}$. Очевидно, $i_{*}$ является эпиморфизмом. Согласно [2], так как $\bar{D}$ и $L_{\infty}$ пересекаются трансверсально, ядро гомоморфизма $i_{*}$ является бесконечной циклической группой, порожденной простым обходом вокруг бесконечно удаленной прямой. Обозначим эту образующую ядра $\operatorname{ker} i_{*}$ через $\gamma_{\infty}$. Так как $i_{*}$ является эпиморфизмом, то ограничение $j: N \rightarrow \bar{N}=[\bar{G}, \bar{G}]$ гомоморфизма $i_{*}$ на $N$ также является эпиморфизмом.

Пусть $f(x, y)=0$ - уравнение $D$ в $\mathbb{C}^{2}$, где $f(x, y)$ - неприводимый многочлен. Многочлен $f(x, y)$ определяет морфизм $F: \mathbb{C}^{2} \rightarrow \mathbb{C}^{1}$, заданный уравнением 
$f(x, y)=z$ так, что $D=F^{-1}(0)$ является нулевым слоем. Рассмотрим ограничение $\varphi: \mathbb{C}^{2} \backslash D \rightarrow \mathbb{C}^{1} \backslash\{0\}=\mathbb{C}^{*}$ морфизма $F$ на $\mathbb{C}^{2} \backslash D$. Индуцированньй этим отображением гомоморфизм $\varphi_{*}: G \rightarrow \pi_{1}\left(\mathbb{C}^{*}\right) \cong \mathbb{Z}$ является эпиморфизмом, так как общий слой морфизма $\varphi$ связен.

$\mathrm{C}$ одной стороны, хорошо известно, что $\pi_{1}\left(\mathbb{C}^{2} \backslash D\right)$ порождается следуюшими образующими, которые ниже мы будем называть геометрическими. По определению геометрическая образующая $\gamma=l^{-1} s l$ - это петля, состоящая из пути $l$, окружности малого радиуса $s$ вокруг $D$ и пути $l^{-1}$, где $l$ соединяет базисную точку фундаментальной группы с точкой $x$, близкой к $D, s$ есть (положительно ориентированная) окружность, лежашая в действительной плоскости, проходяшей через $x$ и пересекающейся трансверсально с $D$ в некоторой точке $y \in D$, являюшейся центром окружности $s$. Если $D$ - неприводимая кривая, то все геометрические образуюшие сопряжены друг другу. Следовательно, естественный эпиморфизм

$$
\alpha: G \rightarrow G / N \simeq H_{1}\left(\mathbb{C}^{2} \backslash D, \mathbb{Z}\right) \simeq \mathbb{Z}, \quad N=[G, G]
$$

отображает все геометрические образуюшие группы $G$ в одну и ту же образующую группы $\mathbb{Z}$. Легко видеть, что $\varphi_{*}$ также отображает все геометрические образуюшие группы $G$ в некоторую образуюшую $\mathbb{Z}$. Поэтому гомоморфизмы $\varphi_{*}$ и $\alpha$ совпадают. Более того, гомоморфизм $\alpha$ позволяет нам рассмотреть $G$ как полупрямое произведение $G \simeq N \ltimes \mathbb{Z}$. Зафиксируем одну из геометрических образуюших, обозначим ее через $\gamma$. Мы можем считать, что $\gamma-$ это образующая второго сомножителя $\mathbb{Z}$ в полупрямом произведении. Тогда $\gamma_{\infty}$ может быть представлено в виде произведения: $\gamma_{\infty}=\nu \gamma^{d}$, где $d=\operatorname{deg} f(x, y)-$ степень кривой $D$ и $\nu$ - некоторьй элемент группы $N$. Так как пересечение $\operatorname{ker} i_{*} \cap N$ тривиально, то гомоморфизм $j: N \rightarrow \bar{N}$ является изоморфизмом.

2. ДоказАтеЛЬСтво теоремы $2^{\prime}$. Рассмотрим отображение $F$, определенное многочленом (2), и обозначим через $X=\mathbb{C}^{2} \backslash D$ дополнение к $D$. Хорошо известно, что существует конечное множество точек $\left\{z_{1}, \ldots, z_{n}\right\} \subset \mathbb{C}^{*}$ такое, что

$$
F: X \backslash F^{-1}\left(\left\{z_{1}, \ldots, z_{n}\right\}\right) \rightarrow \mathbb{C}^{*} \backslash\left\{z_{1}, \ldots, z_{n}\right\}
$$

является локально тривиальным $C^{\infty}$-расслоением. Как и в [1], пусть $B_{i}-$ диск с центром в точке $z_{i}$ радиуса $r_{i} \ll 1$, и пусть $\partial B_{i}$ - его граница. Выберем две не совпадаюшие между собой точки $z_{i, 1}, z_{i, 2}$, принадлежащие $\partial B_{i}$. Точки $z_{i, 1}$ и $z_{i, 2}$ делят $\partial B_{i}$ на две дуги, $\gamma_{i, 1}$ и $\gamma_{i, 2}$. Выберем непересекающиеся пути $\gamma_{i}$, соединяющие точки $z_{i, 1}$ и $z_{i+1,2}\left(z_{n+1,2}=z_{1,2}\right)$ и лежащие вне $\cup B_{i}$, и пусть $\gamma_{i, 1}$ - такая дуга границы $\partial B_{i}$, что $l_{i n}=\left(\cup \gamma_{i, 1}\right) \cup\left(\cup \gamma_{i}\right)$ является границей ограниченного множества $V$, содержашего начало координат $o \in \mathbb{C}^{1}$ и такого, что $z_{i} \notin V$ для всех $i$, 
$1 \leqslant i \leqslant n$ (см. рис. 1 в [1]). Пусть $l_{e x}-$ граница множества $V \cup\left(\cup B_{i}\right)$. Положим $T=\left(\cup B_{i}\right) \cup\left(\cup \gamma_{i}\right)$. Множество $Z=F^{-1}(T)$ назьвается ожерельем кривой $D$.

Так как $T$ является ретрактом пространства $\mathbb{C}^{*}$ и расслоение $F: X \backslash Z \rightarrow \mathbb{C}^{*} \backslash T$ локально тривиально, то имеет место следуюшее

ПРЕДЛОЖЕНИЕ 1 [1]. Если D удовлетворяет условию (*), то $X=\mathbb{C}^{2} \backslash D$ и ожерелье $Z$ кривой $D$ гомотопически әквивалентны.

Таким образом, $\pi_{1}\left(\mathbb{C}^{2} \backslash D\right) \simeq \pi(Z)$, и, более того, мы имеем следующую коммутативную диаграмму:

$$
\begin{array}{cc}
\pi_{1}\left(\mathbb{C}^{2} \backslash D\right) & \longleftarrow \sim \pi(Z) \\
F_{*} \downarrow & \uparrow F_{*} \\
\pi_{1}\left(\mathbb{C}^{*}\right) & \longleftarrow \sim \pi_{1}(T) \underset{\sim}{\sim} .
\end{array}
$$

Легко видеть, что если $D$ удовлетворяет условию $(*)$, то $F_{*}$ является эпиморфизмом.

Пусть $z_{0} \in \gamma_{n}$ - обшая точка, и пусть $Y=F^{-1}\left(z_{0}\right)$ - слой над $z_{0}$. Вложение $Y \subset Z$ индуцирует гомоморфизм $\psi: \pi_{1}(Y) \rightarrow \pi_{1}(Z)$. Очевидно, что $\operatorname{Im} \psi \subset \operatorname{ker} F_{*}$. В [1] была доказана следуюшая теорема.

Теорема. Если $D \subset \mathbb{C}^{2}$ удовлетворяет условию (*), то последовательность

$$
\pi_{1}(Y) \stackrel{\psi}{\longrightarrow} \pi_{1}\left(\mathbb{C}^{2} \backslash D\right) \stackrel{F_{*}}{\longrightarrow} \mathbb{Z} \longrightarrow 0
$$

является точной.

СледСТвИЕ 1. Если $D \subset \mathbb{C}^{2}$ удовлетворяет условию $(*)$, то $N=\operatorname{ker} F_{*}$ является конечно порожденной группой.

Обозначим через $Z_{e x}=F^{-1}\left(l_{e x}\right)$ прообраз кривой $l_{e x}$. Вложение $Y \subset Z_{e x} \subset Z$ и морфизм $F$ приводят к следующей коммутативной диаграмме:

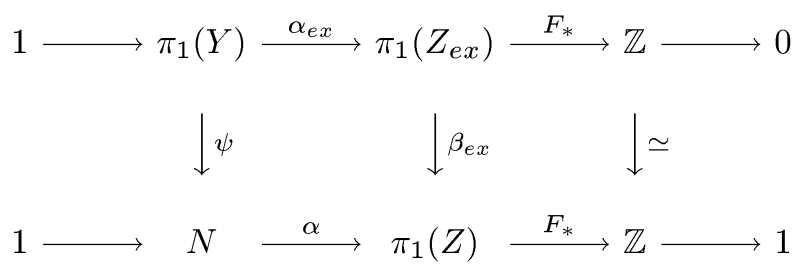

Отображение $F: Z_{e x} \rightarrow l_{e x}$ является локально тривиальньм расслоением. Поэтому все строки в этой диаграмме являются точными.

Обозначим через $h_{e x}$ диффеоморфизм слоя $Y$, индуцированный обходом вдоль $l_{e x}$. 
3. Зафиксируем точку $y_{0} \in Y_{0}=Y$. Пусть $l_{i} \subset l_{e x}$ - путь, соединяюший точку $z_{0}$ с точкой $z_{i, 2}$ (мы используем здесь обозначение из п. 2) и состояший из части пути $\gamma_{n}$ до точки $z_{1,1}$, пути от $z_{1,1}$ до $z_{1,2}$ вдоль $\gamma_{1,2}$, пути $\gamma_{1}$, пути вдоль $\gamma_{2,2}$ из $z_{2,1}$ до $z_{2,2}$ и т.д. до точки $z_{i, 1}$. Если мы зафиксируем локальные тривиализации расслоения $F: Z_{e x} \rightarrow l_{e x}$ над некоторым покрытием петли $l_{e x}$, то пути $l_{i}$ однозначно поднимаются до путей $\bar{l}_{i} \subset Z_{e x}$, начинающихся в точке $y_{0}$. Обозначим через $y_{i}$ конец пути $\bar{l}_{i}$.

Пусть $\bar{B}_{i}=F^{-1}\left(B_{i}\right)$. Выбранные выше пути $\bar{l}_{i}$ определяют гомоморфизмы $\rho_{i}: \pi_{1}\left(\bar{B}_{i}, y_{i}\right) \rightarrow \pi_{1}\left(Z, y_{0}\right)$. Обозначим через $\psi_{i}: \pi_{1}\left(Y_{i}, y_{i}\right) \rightarrow \pi_{1}\left(\bar{B}_{i}, y_{i}\right)$ гомоморфизмы, индуцированные вложениями, где $Y_{i}=F^{-1}\left(z_{i, 1}\right)$. По лемме 2 из [1] гомоморфизмы $\psi_{i}$ являются эпиморфизмами.

Так как $F: Z_{e x} \rightarrow l_{e x}$ является локально тривиальньм $C^{\infty}$-расслоением, то выбранные выше пути $\bar{l}_{i}$ определяют изоморфизмы $\alpha_{i}: \pi_{1}\left(Y_{i}, y_{i}\right) \rightarrow \pi_{1}\left(Y_{0}, y_{0}\right)$. Следовательно, используя эти изоморфизмы, мы можем отождествить группы $\pi_{1}\left(Y_{i}, y_{i}\right)$ с группой $\pi_{1}\left(Y_{0}, y_{0}\right)$ и определить эпиморфизмы $\psi_{i}: \pi_{1}\left(Y_{0}, y_{0}\right) \rightarrow$ $\pi_{1}\left(\bar{B}_{i}, y_{i}\right)$.

4. Отождествим $\pi_{1}\left(l_{e x}\right)$ и $\pi_{1}(T)$, используя изоморфизм, индуцированный вложением $l_{e x} \subset T$. Точная последовательность

$$
1 \longrightarrow N \longrightarrow \pi_{1}\left(Z, y_{0}\right) \stackrel{F_{*}}{\longrightarrow} \pi_{1}\left(T, z_{0}\right) \longrightarrow 1
$$

определяет бесконечное циклическое накрытие $\tilde{g}: \widetilde{Z} \rightarrow Z$, которое может быть включено в коммутативную диаграмму

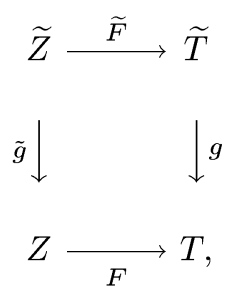

где $g: \widetilde{T} \rightarrow T-$ универсальное накрытие. Выберем точку $\tilde{y}_{0} \in \tilde{g}^{-1}\left(y_{0}\right)$, и пусть $\tilde{z}_{0}=\widetilde{F}\left(\tilde{y}_{0}\right)$. Тогда $\pi_{1}\left(\widetilde{Z}, \tilde{y}_{0}\right)=N$.

Пространство $\widetilde{T}$ является несвязным объединением счетного числа дисков $B_{i, j}$, $j \in \mathbb{Z}$, так что $B_{i, j} \subset g^{-1}\left(B_{i}\right)$. Эти диски соединены последовательно путями (см. рис. 2 в [1]) так, что вместе с этими путями они образуют цепь. Занумеруем диски $B_{i, j}$ в порядке, индуцированном порядком в этой цепи так, как это изображено на рис. 2 в [1]. На каждом пути, соединяюшем соседние диски, выберем по точке $\tilde{z}_{i}$ (на пути, соединяюшем диски $B_{n,-1}$ и $B_{1,1}$, мы берем выбранную вьше точку $\tilde{z}_{0}$ ) 
и занумеруем их в порядке, индуцированном порядком на цепи (так, что точка $\tilde{z}_{0}$ имеет номер 0).

Обозначим через $\widetilde{T}_{k n, m n}$ "часть" пространства $\widetilde{T}$, лежащего между точками $\tilde{z}_{k n}$ и $\tilde{z}_{m n}, m>k$, где $n-$ число дисков $B_{i}$, принадлежаших $T$. Пусть $\widetilde{Z}_{k n, m n}=$ $\widetilde{F}^{-1}\left(\widetilde{T}_{k n, m n}\right)$.

ЛЕмМА 1. $\pi_{1}\left(\widetilde{Z}_{k n, m n}\right)$ является конечно определенной группой.

ДокАЗАтельство. Пусть $\tilde{z}_{0, i, j}$ - центр диска $B_{i, j}$. Рассмотрим пространство

$$
\widetilde{Z}_{k n, m n}^{0}=\widetilde{F}^{-1}\left(\widetilde{T}_{k n, m n} \backslash \bigcup_{i=1}^{n} \bigcup_{j=k}^{m} \tilde{z}_{0, i, j}\right)
$$

Так как расслоения $\widetilde{F}: \widetilde{F}^{-1}\left(B_{i, j} \backslash\left\{\tilde{z}_{0, i, j}\right\}\right) \rightarrow B_{i, j} \backslash\left\{\tilde{z}_{0, i, j}\right\}$ являются локально тривиальными $C^{\infty}$-расслоениями над проколотыми дисками с некомпактными римановыми поверхностями в качестве слоев, то фундаментальные группы $\pi_{1}\left(\widetilde{F}^{-1}\left(B_{i, j} \backslash\left\{\tilde{z}_{0, i, j}\right\}\right)\right)$ являются конечно определенными группами. Применяя теорему Зайферта-ван Кампена, получаем, что $\pi_{1}\left(\widetilde{Z}_{k n, m n}^{0}\right)$ является конечно определенной группой. Прообраз $\widetilde{F}^{-1}\left(\bigcup_{i=1}^{n} \bigcup_{j=k}^{m} \tilde{z}_{0, i, j}\right)$ есть объединение конечного числа римановых поверхностей, и ядро естественного эпиморфизма $\pi_{1}\left(\widetilde{Z}_{k n, m n}^{0}\right) \rightarrow \pi_{1}\left(\widetilde{Z}_{k n, m n}\right)$ порождается геометрическими образующими - простыми обходами вокруг этих поверхностей. Так как для неприводимой римановой поверхности любые две геометрические образующие сопряжены друг другу, то $\pi_{1}\left(\widetilde{Z}_{k n, m n}\right)$ является конечно определенной группй.

5. Лемма 2. Если $f(x, y)-$ приведенный многочлен, $\bar{D}$ и $L_{\infty}$ пересекаются трансверсально, то $h_{e x}^{d}=\mathrm{id}$, əде $d=\operatorname{deg} f(x, y)$.

ДокАЗАТЕЛЬСТво. Морфизм $F$ задает рациональное отображение

$$
F: \mathbb{P}^{2}=\mathbb{C}^{2} \cup L_{\infty} \rightarrow \mathbb{P}^{1}=\mathbb{C}^{1} \cup\{\infty\}
$$

Пусть $\sigma: \overline{\mathbb{P}}^{2} \rightarrow \mathbb{P}^{2}$ - композиция $\sigma$-процессов такая, что $\bar{F}=F \cdot \sigma: \overline{\mathbb{P}}^{2} \rightarrow \mathbb{P}^{1}$ является морфизмом.

Пусть $\tilde{f}\left(x_{0}, x_{1}, x_{2}\right)$ - однородный многочлен, соответствующий многочлену $f$, $f(x, y)=\tilde{f}(1, x, y)$. Уравнение $z^{d}=\tilde{f}\left(x_{0}, x_{1}, x_{2}\right)$ определяет нормальную проективную поверхность $\widetilde{X}_{d} \subset \mathbb{P}^{3}$ и морфизм $\tilde{\phi}_{d}: \widetilde{X}_{d} \rightarrow \mathbb{P}^{2}$. Прообраз $\tilde{\phi}_{d}^{-1}\left(L_{\infty}\right)=\bar{Y}_{\infty}$ является неособой кривой. Обозначим через $\bar{X}_{d}$ нормализацию поверхности $\overline{\mathbb{P}}^{2}$ в поле $\mathbb{C}(x, y, z)$ и через $\phi_{d}: \bar{X}_{d} \rightarrow \overline{\mathbb{P}}^{2}$ соответствующий морфизм.

Выберем некоторую окрестность $U$ точки $\infty \in \mathbb{P}^{1}$, изоморфную диску $\Delta=\{u \in$ $\mathbb{C}|| u \mid \leqslant 1\}$ (точка $u=0$ соответствует точке $\infty \in U$ ) и такую, что отображение 
$\bar{F}: \bar{F}^{-1}(U \backslash \infty) \rightarrow U \backslash \infty$ является гладким собственным морфизмом. Положим $\bar{U}=\bar{F}^{-1}(U)$ и $\widetilde{U}=\phi_{d}^{-1}(\bar{U})$. Мы получим следующую коммутативную диаграмму:

$$
\begin{aligned}
& \tilde{U} \stackrel{\phi_{d}}{\longrightarrow} \bar{U} \\
& \downarrow \bar{F}_{d} \quad{ }^{\stackrel{F}{ }} \\
& \Delta \stackrel{\psi_{d}}{\longrightarrow} U,
\end{aligned}
$$

где $\psi_{d}$ определено уравнением $u=v^{d}(v-$ координата в $\Delta)$. Так как прообраз $\bar{F}_{d}^{-1}(0)=\phi_{d}^{-1}\left(L_{\infty}\right)$ является невырожденным слоем расслоения $\bar{F}_{d}$, то монодромия $h_{d}$, действуюшая на обшем слое и определяемая обходом вокруг гранищы диска $\Delta$, тривиальна. С другой стороны, из коммутативной диаграммы (3) следует, что $h_{d}=h_{e x}^{d}$. Лемма 2 доказана.

6. Следующая лемма завершает доказательство теоремы $2^{\prime}$.

ЛЕмма 3. Если $f(x, y)$ и $\bar{D}$ удовлетворяют условиям леммы 2, то $\pi_{1}\left(\widetilde{Z}_{k d n, m d n}\right)$ изоморфны $\pi_{1}\left(\widetilde{Z}_{0, d n}\right)$ для всех $k$ u $m$.

ДокАЗАТЕЛьСтво. Пусть $\tilde{l}_{e x}=g^{-1}\left(l_{e x}\right)$ и $\widetilde{Z}_{e x}=\tilde{g}^{-1}\left(Z_{e x}\right)$. Тогда $\widetilde{F}: \widetilde{Z}_{e x} \rightarrow$ $\tilde{l}_{e x}$ является тривиальным $C^{\infty}$-расслоением. Рассмотрим слои $Y_{s}=\widetilde{F}^{-1}\left(\tilde{z}_{s}\right)$ этого расслоения. Если мы выберем тривиализацию, то мы можем отождествить все эти слои; другими словами, выбранная тривиализация индуцирует диффеоморфизмы $\alpha_{i, j}: Y_{i} \rightarrow Y_{j}$. Если $k d n \leqslant s \leqslant m d n$, то $Y_{s} \subset \widetilde{Z}_{k d n, m d n}$, и это вложение индуцирует эпиморфизм $\psi_{s, k d n, m d n}: \pi_{1}\left(Y_{s}\right) \rightarrow \pi_{1}\left(Z_{k d n, m d n}\right)$ такой, что если $k d n \leqslant r \leqslant m d n$, то $\psi_{s, k d n, m d n} \alpha_{r, s^{*}}=\psi_{r, k d n, m d n}$.

Все пространства $\widetilde{Z}_{k d n,(k+1) d n}, k \in \mathbb{Z}$, естественным образом диффеоморфны друг другу, так как эти пространства являются прообразами $d$-кратных обходов ожерелья $T$, начинаюшихся в точке $z_{0}$. Эти диффеоморфизмы позволяют нам отождествить фундаментальные группы $\pi_{1}\left(\widetilde{Z}_{k d n,(k+1) d n}\right) \simeq \pi$ для всех $k$. Это отождествление согласовано со сделанным отождествлением слоев $Y_{r}$; другими словами, следующая диаграмма является коммутативной:

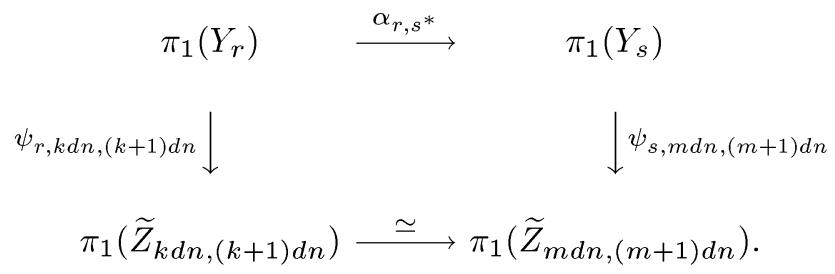

Чтобы получить $\widetilde{Z}_{0,2 d n}$ из $\widetilde{Z}_{0, d n}$ и $\widetilde{Z}_{d n, 2 d n}$ (аналогично для $\left.\widetilde{Z}_{-2 d n, 0}\right)$, мы должны склеить эти два подпространства пространства $\widetilde{Z}_{0,2 d n}$ вдоль диффеоморфных 
слоев $Y_{d n} \subset \widetilde{Z}_{0, d n}$ и $Y_{d n} \subset \widetilde{Z}_{d n, 2 d n}$. Правило склейки определяется монодромией $h_{e x}^{d}$. В нашем случае по лемме 2 монодромия $h_{e x}^{d}=\mathrm{id}$. Применяя теорему Зайферта-ван Кампена, получаем существование изоморфизма $\psi_{0,2}: \pi_{1}\left(\widetilde{Z}_{0, d n}\right) \rightarrow$ $\pi_{1}\left(\widetilde{Z}_{0,2 d n}\right)$, согласованного с эпиморфизмами $\alpha_{*}$. Очевидная индукция завершает доказательство леммы 3 .

\section{Список литературы}

1. Куликов Вик. С. Многочлены Александера плоских алгебраических кривых // Изв. РАН. Сер. матем. 1994. Т. 42. №1. С. 67-90.

2. Nori M. Zariski's conjecture and related problems // Ann. Sci. Ec. Norm. Sup. Ser.4. 1983. V. 16. P. 305-344.

3. Stallings J. R. On fibering certain 3-manifolds // Topology of 3-manifolds. Proc. Top. Inst. Univ. Georgia, 1961; // Englewood Cliffs / Ed. M.K. Fort. N. J.: Prentice Hall, 1962. P. $95-100$.

e-mail: victor@olya.ips.ras.ru

Поступило в редакцию 4.VI.1996 